\title{
Preferencia de tipos de gráficos por parte de productores rurales para comunicación y divulgación técnica
}

Carlos Alberto Naveira

Estación Experimental Agropecuaria Concepción del Uruguay, INTA.

naveira.carlos@inta.gob.ar

ORCID: https://orcid.org/0000-0001-9693-2184

\author{
Aldo Calzolari \\ Instituto Universitario Hospital Italiano Buenos Aires. \\ aldo.calzolari@hospitalitaliano.org.ar \\ ORCID: https://orcid.org/0000-0002-1823-4521 \\ Fecha de finalización: 1 de junio de 2021. \\ Recibido: 8 de agosto de 2021 . \\ Aceptado: 25 de octubre de 2021. \\ DOI: https://doi.org/10.26422/aucom.2021.1002.nav
}

\section{Resumen}

La comprensión de gráficos de datos, tanto a nivel científico como a nivel de divulgación científica, es un tema de creciente interés en el mundo, en particular en el caso de productores rurales, ya que las agencias y otras instituciones deben explicar las mejoras y aportes técnicos en forma sencilla y comprensible. En este trabajo, por medio de una encuesta, se investigó la preferencia por diversos tipos de gráficos a 80 productores rurales de la zona centro-sur de Entre Ríos, Argentina. Se consultó por la preferencia de gráficos de barras (horizontales o verticales) o de líneas (en el mismo gráfico o en gráficos separados). Se obtuvo que prefieren en forma significativa los gráficos de barras por sobre los de líneas $(76,25 \%, \mathrm{p}=0,001)$ y los de barras verticales por sobre los de barras horizontales $(78,75 \%, \mathrm{p}=0,000)$. No hubo diferencia en la preferencia de líneas juntas en el mismo gráfico o separadas en cuatro gráficos. La división por estratos -edad, nivel de estudios o años en la actividad rural- mostraron pequeñas variaciones respecto a los datos globales. En conclusión, los resultados señalan la importancia de diseñar gráficos que sean más sencillos en textos dirigidos a productores rurales.-

Palabras clave: gráficos, figuras, divulgación científica, productor rural, extensión, Argentina.

\section{Rural producers and data graph preferences for communication and technical information}

\begin{abstract}
Understanding data graphs, both when performing scientific work and sharing scientific data, is a topic that is receiving increased attention around the world. This is particularly the case when it comes to rural producers, since agencies and other institutions must explain improvements and technical contributions in a simple, digestible manner. For this paper, we surveyed 80 rural
\end{abstract}


producers in the south-central region of the Province of Entre Ríos in Argentina, investigating their preferences for certain types of data graphs. We asked for their position on bar graphs (either horizontal or vertical) as opposed to line graphs (combined in the same graph or as separate graphs). We found that respondents significantly preferred bar graphs $(76.25 \%, \mathrm{p}=0.001)$ and, among those, also preferred vertical bar graphs over horizontal $(78.75 \%, p=0.000)$. There was no preference for line graphs in a single graph or separated across four graphs. Dividing respondents by age group, academic level, and years of rural activity yielded only slight variations next to the global percentages. In conclusion, these results reveal the importance of designing simpler data graphs when sharing information with rural producers.

Keywords: graphs, figures, scientific communication, rural producers, extension, Argentina.

\section{Preferência de tipos de gráficos por produtores rurais para comunicação e divulgação técnica}

\section{Resumo}

A compreensão de gráficos de dados, tanto no âmbito científico quanto na divulgação científica, é um tema de crescente interesse no mundo, particularmente no caso dos produtores rurais, onde agências e outras instituições devem explicar melhorias e contribuições técnicas em uma maneira simples e compreensível. Neste trabalho, a preferência por diversos tipos de gráficos foi investigada em 80 produtores rurais da zona centro-sul de Entre Ríos, Argentina, por meio de uma pesquisa. Eles foram consultados para a preferência de gráficos de barras (horizontais ou verticais) ou de linhas (no mesmo gráfico ou em gráficos separados). Verificou-se que eles preferem significativamente os gráficos de barras aos gráficos de linhas $(76,25 \%, \mathrm{p}=0,001)$ e os gráficos de barras verticais aos horizontais $(78,75, \mathrm{p}=0,000)$. Não houve diferença na preferência das linhas juntas no mesmo gráfico ou separadas em quatro gráficos. A divisão por estratos de idade, escolaridade ou anos de atividade rural apresentou pequenas variações em relação aos dados globais. Em conclusão, os resultados indicam a importância de se traçar gráficos mais simples em textos dirigidos aos produtores rurais.

Palavras-chave: : gráficos; figuras; divulgação científica; produtor rural; extensão; Argentina.

\section{Introducción ${ }^{1}$}

\section{Estado de situación}

El Instituto Nacional de Tecnología Agropecuaria de Argentina (INTA) cumple un rol muy importante en el país en la generación de conocimiento y en su difusión a productores agropecuarios en todas las áreas de producción primaria. Esta función del INTA se lleva a cabo en cada territorio mediante un sistema de extensión articulado con otras instituciones, como universidades, el Consejo Nacional de Investigaciones Científicas y Tecnológicas (CONICET), asociaciones cooperativas, municipios, agrupaciones de trabajadores rurales y oficinas de Gobiernos nacionales y provinciales.

\footnotetext{
Agradecimientos: algunos de estos resultados formaron parte de la tesis de maestría de Carlos Alberto Naveira en la Universidad Austral. Agradecemos a los profesionales del INTA, Estación Experimental Agropecuaria Concepción del Uruguay, que revisaron las versiones preliminares de la encuesta: Med. Vet. José L. Piccinalli, Ing. Agr. Juan Martín Gange, María Eugenia Munilla, Hernán Ferrari y al Dr. Dante Bueno.
} 


\section{Austral Omunicación \\ Volumen 10, número 2 (Diciembre de 2021): 413-425 ISSN (I) 2313-9129. ISSN (E) 2313-9137}

En la actualidad, el INTA representa un aporte clave para el sector agropecuario, agroalimentario y agroindustrial. La investigación y el desarrollo son los pilares de trabajo que toman cuerpo en el Plan Estratégico Institucional (PEI), en el que se despliega una visión de largo plazo para responder a las demandas de todas las regiones del país.

El INTA es un organismo de vanguardia en el desarrollo agrotecnológico mundial, que está junto al productor y sus necesidades asistiendo a los sectores sociales que merecen atención. De este modo, proyecta sus acciones para alcanzar competitividad, sostenibilidad social y económica con sentido nacional, priorizando la sustentabilidad ambiental de los territorios (Instituto Nacional de Tecnología Agropecuaria [INTA], 2016a). Tiene presencia en todo el territorio nacional y es tenido en cuenta como fuente de información para medios digitales argentinos. Según Spina y Díaz (2020, p. 36), para el abordaje de temáticas agrarias, los diarios argentinos recurren al Gobierno nacional, provincial o local en un porcentaje (35\%) mayor que a laboratorios $(32,5 \%)$, universidades nacionales $(7,5 \%)$ y Gobiernos e instituciones extranjeras (7,5\%). Por lo tanto, este rol del INTA implica también la responsabilidad de sus extensionistas cuando realizan publicaciones dirigidas a productores, muchas de las cuales son luego reproducidas en la prensa del país.

Para cumplir estos cometidos, la institución debe cerrar el ciclo y acercar los resultados de estos esfuerzos a las personas que trabajan en la producción agropecuaria. En ese marco, tiene áreas dedicadas a la comunicación institucional y produce material de difusión en distintos tipos de documentos.

En cuanto a la comunicación, cuenta con publicaciones técnicas: notas de divulgación, artículos, guías y manuales, informes, libros, revistas, videos, audios y noticias. También posee una serie de producciones propias denominada Ediciones INTA, que incluye distintas colecciones, catálogos, libros electrónicos, una biblioteca digitalizada, puntos de venta (Librerías INTA) y la Revista RIA (INTA, 2021).

A nivel gráfico, existen publicaciones organizadas en las Bibliotecas INTA. Estos Centros Documentales están

[...] especializados en ciencias agropecuarias y otras disciplinas relacionadas, facilitan el
acceso a la información a la comunidad investigadora interna-externa y a la sociedad en
general, proporcionando apoyo en la formación y difusión del conocimiento. A su vez,
mantienen características propias en cuanto a la especificidad temática de cada región y
cuentan con colecciones de libros, publicaciones periódicas y seriadas, de edición propia
y extra-institucional, conformando así valiosos acervos locales que dan lugar a una red
de Centros Documentales que basan sus líneas de acción en la integración de procesos y
recursos. (INTA, 2016b, p. 1)

Esta actividad se realiza mediante jornadas, congresos, charlas y publicaciones específicas. Sin desmerecer el rol que poseen las primeras en la difusión del conocimiento, tienden a ser efímeras y de localización más dificultosa. Lo que perdura en el entorno digital son las publicaciones técnicas y científicas. 
Carlos Alberto Naveira, Aldo Calzolari

Preferencia de tipos de gráficos por parte de productores rurales para comunicación y divulgación técnica

\section{Divulgación de la ciencia y figuras}

En el ámbito agropecuario, Leach (2017) sostiene una política estatal que incluye la extensión, para "[...] reducir la brecha de conocimiento agrícola al educar a personas de todas las edades en áreas de agricultura y aumentar su participación comunitaria" (p. 3). En cuanto a esto, estudió cómo debe ser la extensión para llegar a esos lugares donde se necesita información y los métodos de comunicación más efectivos. Encontró que los medios mixtos digitales (gráficos, videos y audios digitales) son los que mejor se comprenden. En el INTA se utilizan todos estos recursos.

Dentro de la producción gráfica, las figuras -ya sean gráficos o imágenes- aportan un significativo nivel de comprensión de los datos que se suministran.

Retrospectivamente, las figuras comenzaron a utilizarse casi desde el inicio de la comunicación científica, con el papel en China y con el papiro en Egipto, describiendo fundamentalmente procesos médicos, instrumentos y funciones fisiológicas (Arribalzaga et al., 2005).

En la Argentina, Brunetti (2013) recoge antecedentes del año 1908 que estaban en manos de Francisco Lavalle, publicados en una crítica del diario La Nación, en la que Lavalle expone la relevancia de la comunicación de la ciencia, "escribiendo su preocupación por la resistencia de algunos miembros de la comunidad científica para la vulgarización o divulgación de la ciencia" (p. 140). Además, rescata la idea de "una escritura literaria", signo del trabajo de "vulgarización, como esfuerzo para que muchos conocimientos, lleguen al público en general” (p. 141).

Desde las primeras revistas científicas de la historia, el Journal des sçavans (Francia) y el Philosophical Transactions of the Royal Society (Inglaterra) -ambos publicados por primera vez en 1665 (Arribalzaga et al., 2005)-, las figuras han tenido un desarrollo sostenido y un aumento creciente en complejidad. En ocasiones, llegan a niveles sofisticados de representación gráfica, cuya comprensión a veces resulta dificultosa hasta para especialistas en los campos disciplinares, por ejemplo, las figuras múltiples de revistas de alto nivel como Nature o Science.

El empleo de figuras que contienen gráficos se ha tornado un elemento crucial. Numerosos estudios señalan la relevancia de utilizar fotografías, gráficos e infografías (Krum, 2013; Palacio Montes, 2017). La comunicación con figuras es beneficiosa para la extensión rural, tal como lo expresa Kassie Waller (2018): “[...] las comunicaciones visuales no solo son beneficiosas para el campo de la educación, sino que también pueden ayudar a los profesionales de la ciencia en sus esfuerzos por compartir investigaciones e ideas complejas del sector agropecuario" (p. 8).

Pero los formatos de visualización no evolucionaron al mismo ritmo que los softwares para preparar gráficos ni las infografías. Más allá de algunos avances limitados (Osinska, 2018; Tufte, 2007), es todavía un tema en discusión y falto de estudios, el cual requiere investigación por contener lagunas metodológicas (Hartley et al., 2013). La 


\section{Austral Omunicación \\ Volumen 10, número 2 (Diciembre de 2021): 413-425 ISSN (I) 2313-9129. ISSN (E) 2313-9137}

sistematización de figuras provista por Tufte (2007) ha hecho importantes contribuciones. Estas innovaciones están siendo gradualmente incorporadas al uso corriente de las personas responsables de comunicación institucional.

En el caso específico del INTA, sus profesionales están abocados a un doble rol: como investigadores/as del sistema científico-técnico del país y como extensionistas, esto es, en la divulgación de conocimientos para el mejoramiento de la actividad agropecuaria. Este doble rol de escribir para el sector científico, por un lado, y para el sector productivo, por otro (que podría no poseer formación científica específica), hace que, en ocasiones, el discurso comunicacional se torne difícil.

Durante el desarrollo de la tesis de maestría de uno de los autores (Naveira, 2019), se realizó una encuesta a productores rurales, orientada a conocer aspectos comunicacionales de datos científicos dirigidos al sector productivo que, para nuestro conocimiento, no se encuentra estudiado en la Argentina. Dentro de esa encuesta se abordaron dos temas: uno sobre comprensión de figuras ${ }^{2}$ y otro destinado a conocer preferencias de figuras.

El objetivo de este artículo es conocer la preferencia de los productores rurales por diversos tipos de figuras: barras (verticales u horizontales), de líneas (juntas o separadas) y de barras versus líneas.

\section{Materiales y métodos}

Se realizó una investigación de tipo cualitativa documental para la búsqueda, análisis y modificación de figuras y una investigación prospectiva cuantitativa mediante una encuesta.

\section{Datos generales de la encuesta y análisis}

Se diseñó una encuesta consistente en 22 preguntas, las primeras 19 dirigidas a conocer detalles de comprensión e interpretación de figuras y las últimas 3 orientadas a conocer las preferencias en cuanto al formato de los gráficos. La encuesta fue revisada en dos instancias por colegas del INTA y, luego de cada una, se hicieron modificaciones. En una tercera instancia fue revisada por tres productores.

Se encuestó a 80 personas provenientes del territorio de influencia del INTA Estación Experimental Agropecuaria Concepción del Uruguay, que cubre los departamentos del sureste de Entre Ríos: San Salvador, Colón, Villaguay, Rosario del Tala, Uruguay, Gualeguaychú, Islas del Ibicuy y el Delta entrerriano (zonas bajas e insulares de Gualeguay, Victoria y Diamante). Luego de obtener el consentimiento informado

Sobre este tema, se encuentra en elaboración un artículo. 
Carlos Alberto Naveira, Aldo Calzolari

Preferencia de tipos de gráficos por parte de productores rurales para comunicación y divulgación técnica

de cada participante, se realizó la encuesta. La información personal se mantuvo en reserva por medio de la codificación de las encuestas.

Los datos se analizaron en conjunto y mediante una segmentación en dos grupos. Así, el total de encuestas se dividió por nivel de estudio, edad y años en la actividad. Dada la elevada cantidad de personas de género masculino con respecto al femenino, se optó por no realizar un análisis segmentado por género.

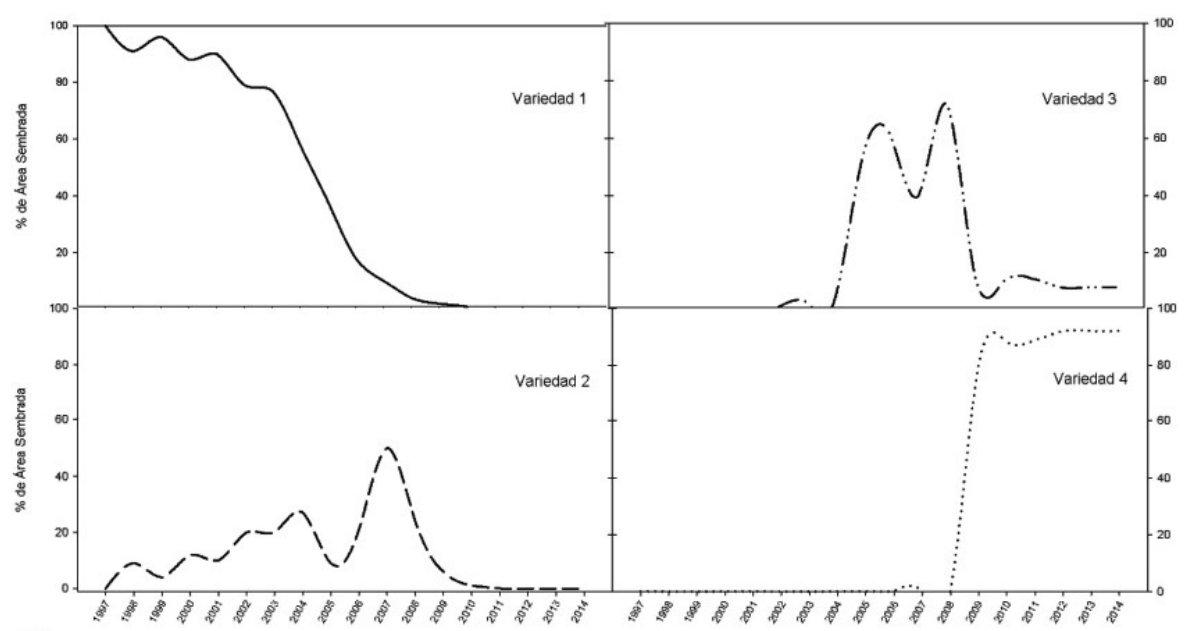

a

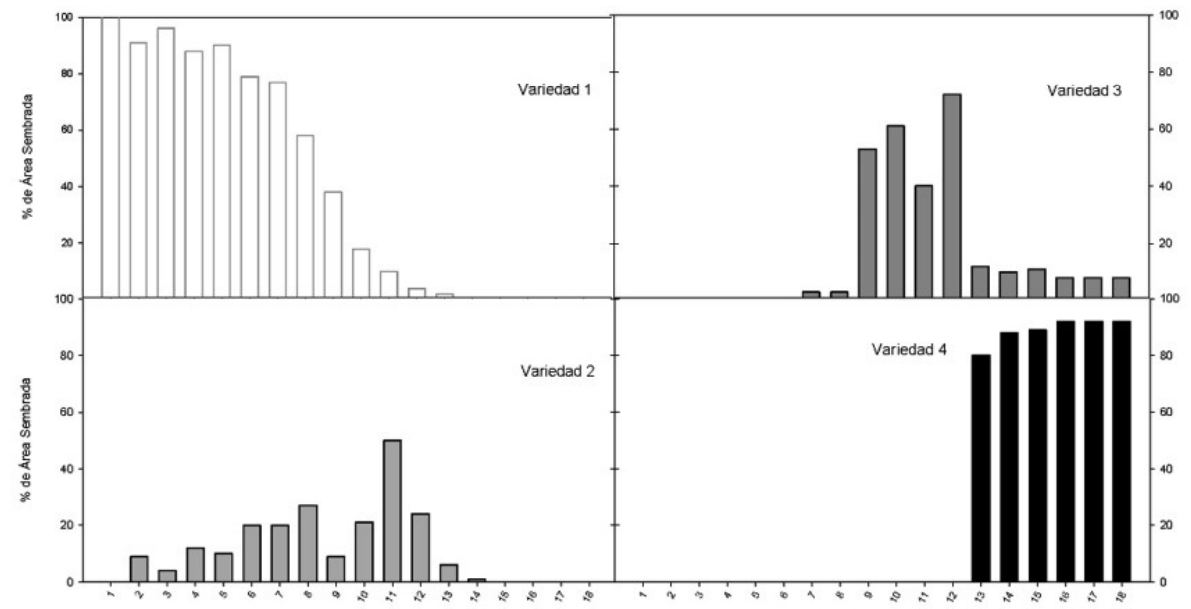

b

Figura 1. Comparación de preferencias por gráficos de líneas (a) o de barras (b). 
Austral Comunicación

Volumen 10, número 2 (Diciembre de 2021): 413-425

ISSN (I) 2313-9129. ISSN (E) 2313-9137

\section{Elaboración de figuras}

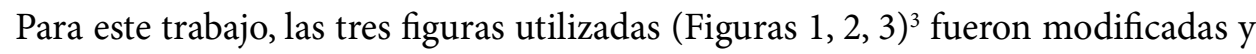
anonimizadas a partir de ejemplos reales de publicaciones científicas argentinas del ámbito agropecuario, con base en las consideraciones de Kelleher y Wagener (2011).

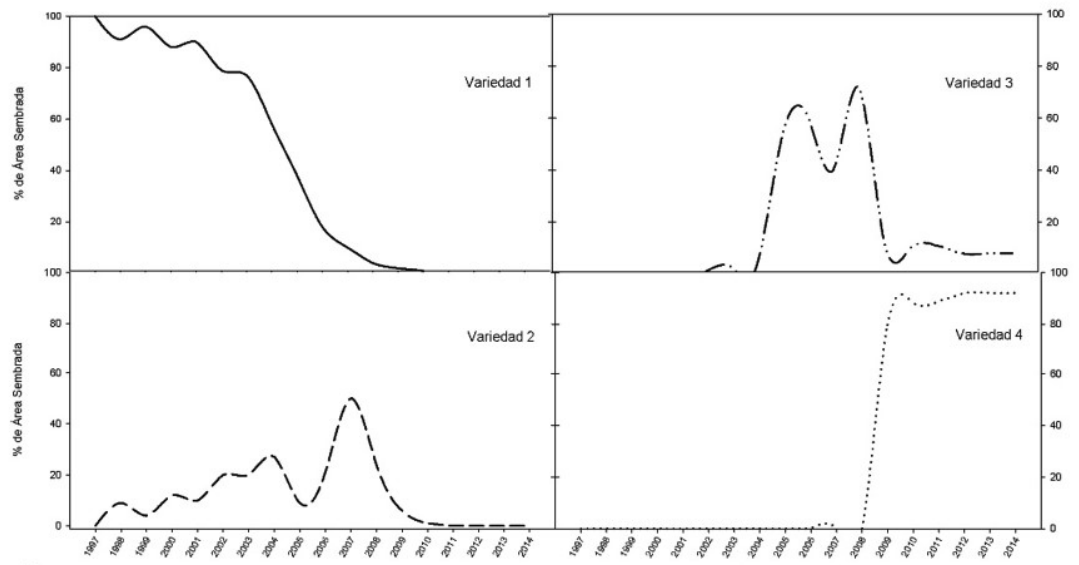

a

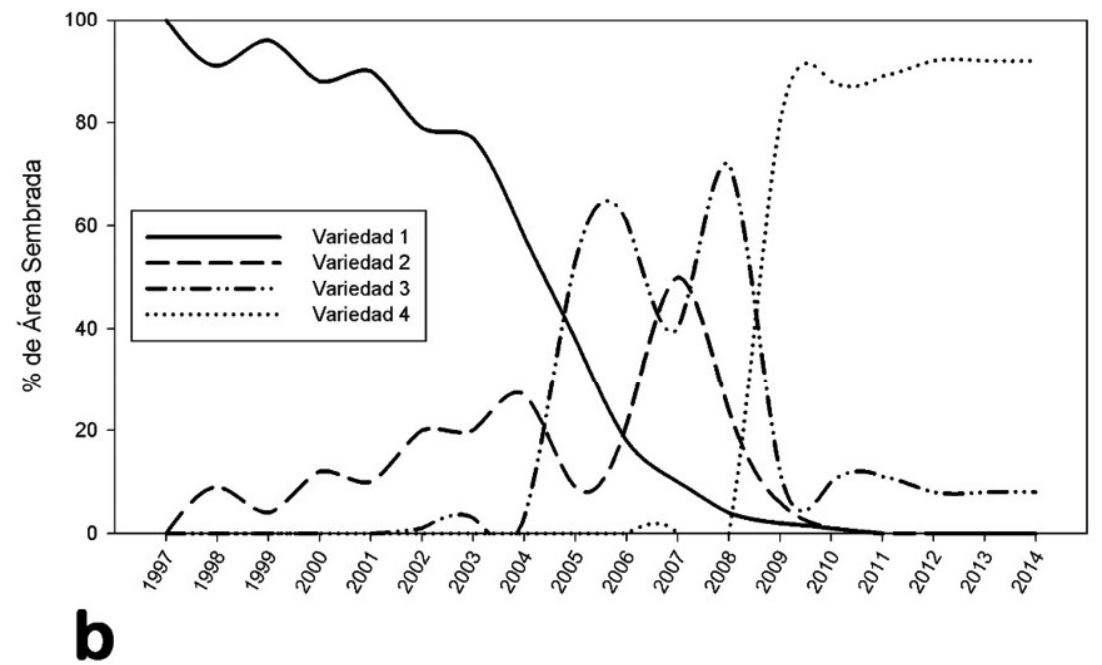

Figura 2. Comparación de preferencias por gráficos de líneas separadas una en cada gráfico (a) o todas juntas en uno (b).

En el trabajo de campo, las figuras fueron presentadas en formato papel a color y se transformaron a escala de grises para la publicación impresa. 

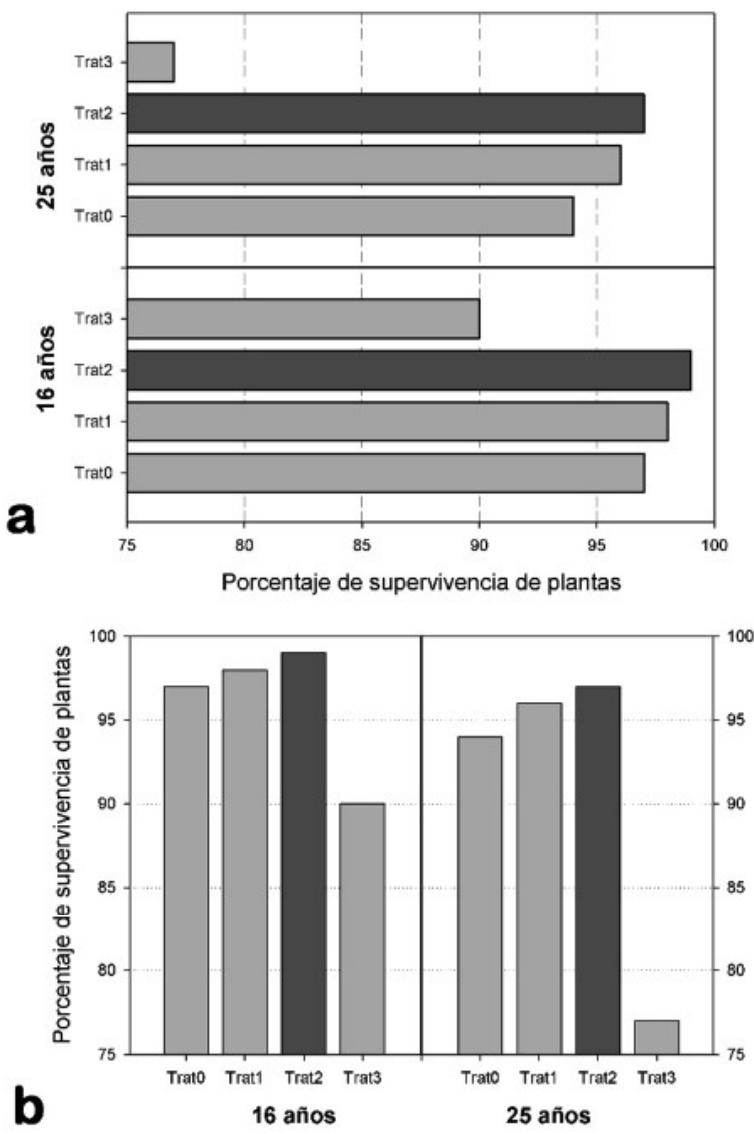

Figura 3. Comparación de preferencias por gráficos de barras horizontales (a) 0 verticales (b).

\section{Análisis estadístico}

Los datos se analizaron mediante el test de Chi². El nivel de significación se estableció en 0,05 .

\section{Resultados}

Las características demográficas de las personas encuestadas (género, nivel educativo, edad y años en la actividad) se muestran en la Tabla 1. A excepción del género, las otras categorías fueron divididas en dos subcategorías, tomando en cuenta criterios internacionales. 
Austral Comunicación

Volumen 10, número 2 (Diciembre de 2021): 413-425

ISSN (I) 2313-9129. ISSN (E) 2313-9137

Tabla 1. Datos demográficos de las 80 personas encuestadas

\begin{tabular}{|c|c|c|c|c|}
\hline & & & $\mathbf{N}$ & $\%$ \\
\hline \multicolumn{5}{|l|}{ Género } \\
\hline \multicolumn{3}{|c|}{ Masculino } & 63 & 78,75 \\
\hline \multicolumn{3}{|c|}{ Femenino } & 17 & 21,25 \\
\hline \multicolumn{5}{|c|}{ Nivel educativo } \\
\hline \multicolumn{3}{|c|}{ No Universitario } & 34 & 42,50 \\
\hline \multicolumn{3}{|c|}{ Agrotécnico } & 14 & \\
\hline \multicolumn{3}{|c|}{ Secundario } & 13 & \\
\hline \multicolumn{3}{|c|}{ Primario } & 7 & \\
\hline \multicolumn{3}{|c|}{ Universitario } & 46 & 57,50 \\
\hline \multicolumn{5}{|l|}{ Edad } \\
\hline \multicolumn{3}{|c|}{ Hasta 55 años } & 55 & 68,75 \\
\hline \multicolumn{3}{|c|}{ Más de 55 años } & 25 & 31,25 \\
\hline \multicolumn{5}{|c|}{ Años en la actividad rural } \\
\hline \multicolumn{3}{|c|}{ Hasta 25 años } & 44 & 55 \\
\hline \multicolumn{3}{|c|}{ Más de 25 años } & 36 & 45 \\
\hline \multicolumn{5}{|c|}{ Principales actividades rurales de las personas encuestadas* } \\
\hline \multicolumn{4}{|c|}{\begin{tabular}{ll|l} 
Ganadería & 43 & Extensionistas
\end{tabular}} & 21 \\
\hline Agricultura & 14 & Apicultores & & 6 \\
\hline Avicultura & 11 & Investigadores & & 4 \\
\hline Horticultura & 11 & Comerciantes & & 3 \\
\hline
\end{tabular}

Los resultados sobre preferencia de formato se muestran en la Tabla 2.

\begin{tabular}{lcl}
\hline & $\%$ & $\mathbf{p}$ \\
\hline $\begin{array}{l}\text { Prefiere gráfico de barras antes que } \\
\text { de líneas }\end{array}$ & 76,25 & 0,001 \\
$\begin{array}{l}\text { Prefiere líneas juntas en un solo } \\
\text { gráfico }\end{array}$ & 58,75 & NS \\
Prefiere gráfico de barras verticales & 78,75 & 0 \\
\hline
\end{tabular}

Tabla 2. Resultados de la aplicación de la encuesta sobre la preferencia de figuras $(n=80)$

Hubo una preferencia significativa por los gráficos de barras por sobre los de líneas y, dentro de los de barras, casi el 80 \% eligió las barras verticales por sobre las horizontales. 
Carlos Alberto Naveira, Aldo Calzolari

Preferencia de tipos de gráficos por parte de productores rurales para comunicación y divulgación técnica

En cambio, la presentación de los datos en gráficos de líneas (un gráfico para cada línea de dato o las cuatro líneas en un mismo gráfico) señaló una ligera preferencia por este último, sin significación estadística (Tabla 2).

El análisis de los resultados de la encuesta segmentado por estudios, edad y años en la actividad (Tabla 3) mostró en todos los estratos preferencia por el gráfico de barras respecto al de líneas. El segmento años en actividad mostró significación estadística en ambos estratos, en tanto que en el segmento de estudios hubo significación estadística para el grupo de personas con nivel universitario y para menos de 25 años en la actividad rural.

Tabla 3. Resultados de la aplicación de la encuesta sobre la preferencia de figuras en los estratos estudiados

\begin{tabular}{lllllll}
\hline Preferencia & \multicolumn{2}{l}{ Estudios } & \multicolumn{2}{l}{ Edad (años) } & \multicolumn{2}{l}{ Actividad (años) } \\
\hline \multirow{2}{*}{ Gráfico de barras antes que el de líneas } & No univ & Univ & 255 & $>55$ & $\geq 25$ & $>25$ \\
\cline { 2 - 8 } Líneas juntas en un solo gráfico & 57,7 & $82,6^{*}$ & $78,2^{*}$ & 72 & $77,3^{*}$ & $75,0^{*}$ \\
Gráfico de barras verticales & 50 & 65,2 & 52,7 & 72 & 52,3 & 66,7 \\
n & $79,4^{*}$ & $78,3^{*}$ & $78,2^{*}$ & $80,0^{*}$ & $79,6^{*}$ & $77,8^{*}$ \\
\hline
\end{tabular}

$\left.{ }^{*}\right)$ : Diferencias significativas $\mathrm{p}<0,05$ por $\mathrm{Chi}^{2}$.

En relación con la preferencia de las personas encuestadas respecto a la forma de presentación de los datos en líneas juntas o separadas, ninguno de los segmentos analizados mostró diferencias estadísticas. No obstante, algunos tuvieron porcentajes de preferencia mayor hacia las líneas juntas en un solo gráfico.

Por último, el análisis de la forma de presentación de datos en forma de barras horizontales o verticales dio una preferencia significativa por las verticales en todos los segmentos y estratos (Tabla 3 ).

\section{Discusión}

Tres estudios previos (Ali y Peebles, 2013; Peebles y Ali, 2009; 2015) demostraron que las personas expertas no tienen inconvenientes para interpretar correctamente los gráficos de barras o de líneas, en tanto que usuarios no expertos obtuvieron peores resultados con los gráficos de líneas en comparación con gráficos de barras equivalentes. Estos autores recomendaron que los gráficos de barras se empleen en los casos en que el objetivo sea una interpretación precisa, destinada a una audiencia general de usuarios que incluya tanto a novatos como a expertos. En la encuesta realizada en este trabajo, hubo una fuerte preferencia por los gráficos de barras respecto a los de líneas (Tabla 2).

Si se consideran "personas expertas" a quienes tienen estudios universitarios y "no expertas" a quienes no los tienen, los datos presentados en este artículo difieren de lo expresado por Ali y Peebles (2013). Por un lado, quienes tienen estudios universitarios 


\section{Austral @municación \\ Volumen 10, número 2 (Diciembre de 2021): 413-425 ISSN (I) 2313-9129. ISSN (E) 2313-9137}

prefieren en forma significativa los gráficos de barras por sobre los de línea. Por el otro, en el caso de las personas sin estudios universitarios, no se evidenciaron diferencias significativas, más allá de que hubo una mayoría que mostró preferencia por gráficos de barras (68\%) respecto a gráficos de líneas (32\%). De los resultados de este trabajo surge la recomendación de utilizar gráficos de barras en los casos en que el objetivo comunicacional sea la interpretación precisa de datos por parte de productores rurales. Esta sugerencia puede extenderse a cualquier público novato de otras áreas disciplinares.

De modo similar a la comparación de gráficos de líneas con los de barras, los productores rurales encuestados prefirieron los gráficos de barras verticales por sobre los de barras horizontales (Tabla 2). No hemos encontrado estudios previos respecto a esta preferencia en productores rurales, lo que constituye otro aporte novedoso. Cox (2004) señala que la selección de barras horizontales o verticales es un tema de gusto personal, aunque, en general, las barras horizontales hacen más fácil identificar los nombres o los rótulos de las categorías y evitan que los gráficos verticales con valores muy elevados queden estéticamente desagradables. En el mismo sentido, Tufte (2007) agrega que los gráficos de barras horizontales son más convenientes que los de barras verticales debido a que el ojo humano está naturalmente más entrenado para detectar desviaciones en el horizonte. Es interesante el contraste entre lo que expresan esas investigaciones y lo que mostraron las encuestas de este trabajo. Una posible explicación es que las investigaciones teóricas mencionadas (Cox, 2004; Tufte, 2007) se refieren a personas entrenadas en visualización de gráficos, mayormente científicos, en tanto que en este estudio la población está orientada a personas que trabajan en producción agropecuaria, sin formación específica en ciencias. En un estudio más antiguo, Zacks y Tversky (1999) encontraron que los gráficos con barras horizontales aparecen mayormente en revistas no científicas y que presentan una tendencia a disminuir en el periodo estudiado (de 1985 a 1994).

El análisis de preferencia entre gráficos de líneas -una en cada gráfico o las cuatro juntas en una misma figura- dio 59\% de preferencia para las líneas separadas (Tabla 2). No obstante, esta diferencia no arrojó diferencias significativas. Zacks y Tversky (1999) encontraron que los gráficos de líneas y puntos son mucho más frecuentes que los de barras en publicaciones científicas, en tanto que en revistas no científicas y periódicos, el 50\% de los gráficos son de líneas y aproximadamente el 50\% son de barras.

De este trabajo surgen interesantes líneas para continuar las investigaciones. Por un lado, ahondar más en el nivel de preferencias y de comprensión de productores rurales por el tipo de gráfico, con incorporación de gráficos de tortas, líneas simples e infografías. Por otro, analizar si hay diferencias cuando las figuras se preparan para la comprensión de datos o para la toma de decisiones a partir de esos datos. 
Carlos Alberto Naveira, Aldo Calzolari

Preferencia de tipos de gráficos por parte de productores rurales para comunicación y divulgación técnica

\section{Conclusiones}

Hay diferencia significativa en la preferencia de productores rurales hacia los gráficos de barras respecto a los de líneas, y hacia los gráficos de barras verticales respecto a los de barras horizontales.

No hay diferencia significativa en la preferencia de los productores rurales por aquellos de líneas juntas (en un mismo gráfico) versus los de líneas separadas (una en cada gráfico).

\section{Referencias}

Ali, N. y Peebles, D. (2013). The effect of gestalt laws of perceptual organisation on the comprehension of three-variable bar and line graphs. Human Factors, 15,183-203. https://doi.org/10.1177/0018720812452592.

Arribalzaga, E., Borracci, R., Giulano, R. y Jacovella, P. (2005). El artículo cientifico: del papiro al formato electrónico. Magister Eos.

Brunetti, P. M. (2013). Visitando las estrellas: Martín Gil y el discurso de divulgación astronómica en la Argentina de comienzos del siglo XX. Austral Comunicación, 1(2), 137-154. https://doi.org/10.26422/aucom.2012.0102.bru.

Cox, N. C. (2004). Speaking Stata: graphing categorical and compositional data. The Stata Journal, 4(2):190-215.

Hartley, J., Cabanac, G., Kozac, M. y Hubert, G. (2013). Research on tables and graphs in academic articles. Pitfalls and promises. Journal of the American Society for Information Science and Technology, 22, 1-7.

Instituto Nacional de Tecnología Agropecuaria. (2016a). Historia del INTA. www.inta. gob.ar/sobre-el-inta/historia.

Instituto Nacional de Tecnología Agropecuaria. (2016b). Bibliotecas Central INTA. www.inta.gob.ar/publicaciones/bibliotecas-inta.

Instituto Nacional de Tecnología Agropecuaria. (2021). ¿Qué es el INTA? www.inta. gob.ar/queeselinta\#.

Kassie Waller, B. S. (2018). The effect of infographics on recall of information about genetically modified foods (Tesis de maestría). Texas Tech University, Texas, Estados Unidos. https://ttu-ir.tdl.org.

Kelleher, C. y Wagener, T. (2011). Ten guidelines for effective data visualization in scientific publications. Environmental. Modelling y Software, 26(6), 822-827. https://doi.org/10.1016/j.envsoft.2010.12.006.

Krum, R. (2013). Cool infographics. Effective communications with data visualization and design. Wiley.

Leach, M. J. (2017). Determining effective communication strategies used by Texas AyM AgriLife Extension to educate the uninformed, uninvolved public (Tesis de maestría). West Texas AyM University, Texas, Estados Unidos. 
Austral Comunicación

Volumen 10, número 2 (Diciembre de 2021): 413-425 ISSN (I) 2313-9129. ISSN (E) 2313-9137

Naveira, C. A. (2019). Optimización de figuras y gráficos para su uso en divulgación cientifica a productores rurales (Tesis de maestría en Gestión de Contenidos). Universidad Austral, Argentina. https://riu.austral.edu.ar/handle/123456789/700.

Osinska, V. (2018). Visualizing the scientific information nowadays: the problems and challenges. Informatio et Scientia, 1(1), 17-25.

Palacio Montes, J. A. (2017). Lectura e interpretación de gráficos estadísticos como estrategia de enseñanza aprendizaje en ciencias (Tesis de maestría). Universidad Nacional de Colombia, Manizales, Colombia. www.unal.edu.co.

Peebles, D. y Ali, N. (2009). Differences in comprehensibility between three-variable bar and line graphs. En Taatgen, N. A. y Van Rijn, H. (Eds.), Proceedings of the Thirty-first Annual Conference of the Cognitive Science Society (pp. 2938-2943). Cognitive Science Society.

Peebles, D. y Ali, N. (2015). Expert interpretation of bar and line graphs: the role of graphicacy in reducing the effect of graph format. Frontiers in Psychology, 6, 1673. https://doi.org/10.3389/fpsyg.2015.01673.

Spina, G. D. y Díaz, C. B. (2020). La cobertura de las noticias científicas en diarios digitales argentinos (2017-2018). Austral Comunicación, 9(1), 5-43. https://doi. org/10.26422/aucom.2020.0901.spi.

Tufte, E. R. (2007). The Visual display of quantitative information (2 ${ }^{\mathrm{a}}$ ed.). Graphics Press.

Zacks J. y Tversky, B. (1999). Bars and lines: A study of graphic communication. Memory \& Cognition, 27, 1073-1079. 